\title{
Supporting Information: Local Confinement Controls Diffusive Nanoparticle Dynamics in Semidilute Polyelectrolyte Solutions
}

\author{
Ali H. Slim, ${ }^{\dagger}$ Ryan Poling-Skutvik, ${ }^{\ddagger}$ and Jacinta C. Conrad ${ }^{*} \dagger$ \\ $\dagger$ Department of Chemical and Biomolecular Engineering, University of Houston, Houston, \\ $T X 77204-4004$ \\ $\ddagger$ Department of Chemical and Biomolecular Engineering, University of Pennsylvania, \\ Philadelphia, PA 19104 \\ E-mail: jcconrad@uh.edu
}

\section{Intrinsic Viscosity}

Dilute aqueous sodium polystyrene sulfonate (NaPSS) solutions were prepared by diluting a concentrated stock solution. Samples were introduced into a glass capillary viscometer (Cannon Instrument Company, 0C), which was submerged into a water bath for better temperature control. Although the capillary viscometer operates at a high shear rate of approximately $300 \mathrm{~s}^{-1}$, we observe excellent agreement between the viscosities determined using the viscometer and the rheometer (Figure 1 in manuscript), as evident by the consistent crossover between dilute and semidilute regimes at the overlap concentration calculated using capillary viscometer. These observations suggest that the viscometer accurately captures NaPSS contribution in dilute solutions. Figure S1 shows the sample viscosity as a function of NaPSS concentration. We fitted the data to the two term virial expansion of viscosity $\eta=\eta_{0}\left(1+[\eta] C_{\mathrm{NaPSS}}\right)$, where $[\eta]$ is the intrinsic viscosity, and $\eta_{0}$ is the solvent viscosity. 
Using the fitting equation, we determined the intrinsic viscosity $[\eta]$. We then calculated the overlap concentration $\left(c^{*}=1 /[\eta]\right) 0.13,0.41$, and $2.1 \mathrm{~g} / \mathrm{L}$ and determined the radius of gyration $\left(R_{\mathrm{g}, 0}=\left(M_{\mathrm{w}} /\left(4 / 3 \pi N_{\mathrm{av}} c^{*}\right)\right)^{1 / 3}\right) 190,130$, and $75 \mathrm{~nm}$ for $2200 \mathrm{kDa}$ polymer at $10^{-6}, 10^{-3}, 10^{-1} \mathrm{M}$ ionic strength, respectively. Flory exponent $\nu$ at each ionic strength was determined from the scaling of polymer size as a function of molecular weight (Figure S2) according to the Flory theory of fractal chains. ${ }^{1}$
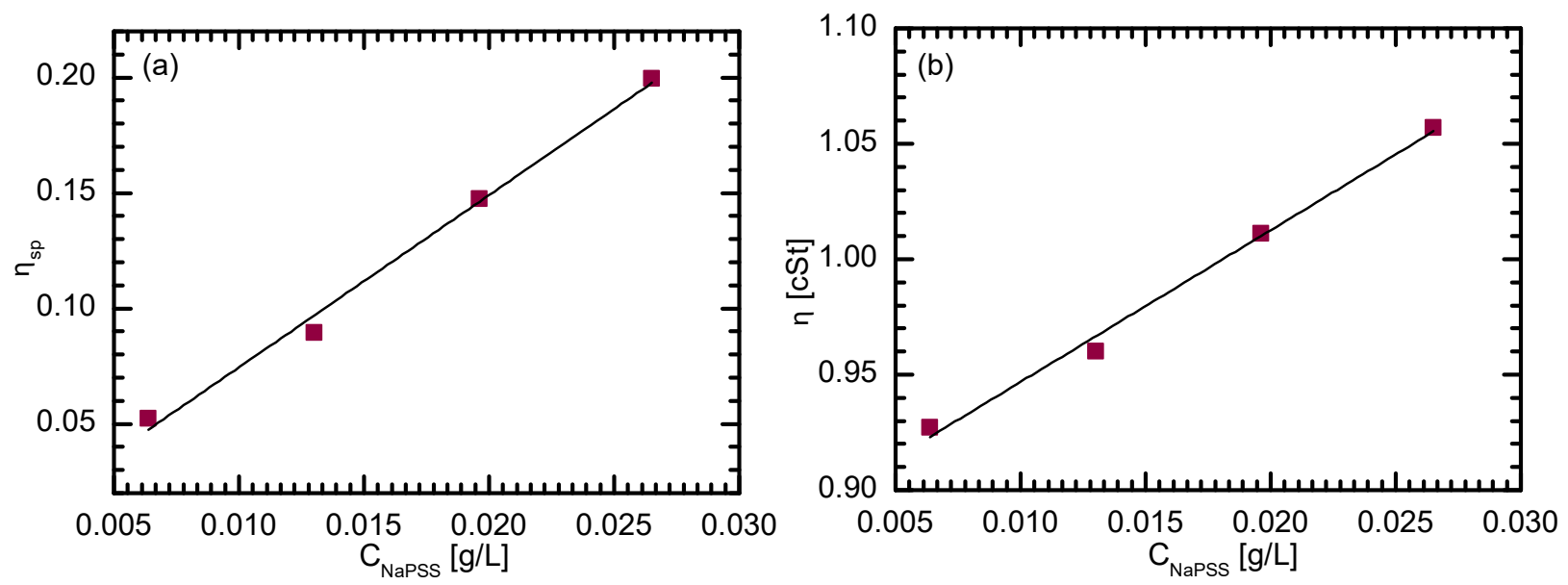

Figure S1: Specific $\eta_{\mathrm{sp}}$ (a) and solution viscosity $\eta$ (b) as a function of NaPSS concentration $C_{\mathrm{NaPSS}}$ for $2200 \mathrm{kDa}$ NaPSS in $10^{-6} \mathrm{M}$ solutions.

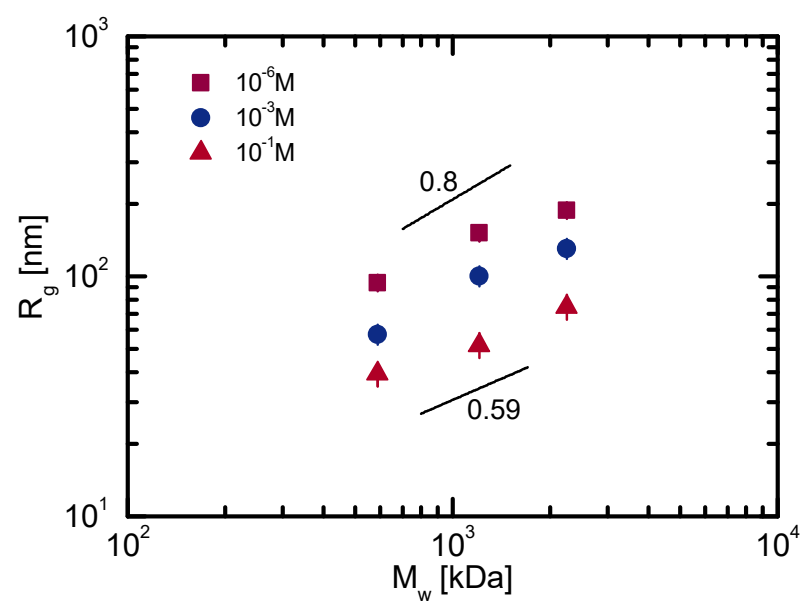

Figure S2: Radius of gyration $R_{\mathrm{g}}$ as a function of polymer molecular weight $M_{\mathrm{w}}$. Solid lines represent scaling at the low and high salt limits for polyelectrolytes. 


\section{Polymer Length Scales}

Sodium polystyrene sulfonate is a polyelectrolyte with one charged functional group per repeat unit. Charge repulsion leads to persistence length values larger than those of neutral polymers. The chains, however, remain flexible at length scales larger than persistence length due to their large length. This causes chains to adopt a semiflexible conformation. Therefore, the scaling behavior of the physical properties of sodium polystyrene sulfonate in salt free conditions is between neutral polymers in good solvent and polyelectrolytes with no salt. We calculated the contour length $l=N a$, where $N$ is the number of repeat units, and $a$ is the length of a carbon bond $(1.54 \AA)$. Polymer end-to-end distance was determined using $R_{\mathrm{e}}=\sqrt{N} a$. Persistence length $l_{\mathrm{p}}$ was estimated to be equal to the Debye screening length $k_{\mathrm{D}}=1 / \sqrt{4 \pi i}$, where $i$ is the ion density. We calculated the Kuhn length to be twice the persistence length. Correlation length $\xi$ and polymer size $R_{\mathrm{g}}$ beyond the dilute regime were calculated via $\xi=R_{\mathrm{g}, 0}\left(c / c^{*}\right)^{\frac{-\nu}{3 \nu-1}}$ and $R_{g}=R_{\mathrm{g}, 0}\left(c / c^{*}\right)^{-\frac{\nu-0.5}{3 \nu-1}}$.

Table S1: Estimations of relevant length scales for $2200 \mathrm{kDa}$ NaPSS in dilute solutions at $10^{-6} \mathrm{M}$ ionic strength.

\begin{tabular}{|c|c|c|c|}
\hline Polymer Property & $10^{-6}$ M Solution & $10^{-3}$ M Solution & $1^{-1}$ M Solution \\
\hline Radius of gyration $R_{\mathrm{g}}$ & $190 \mathrm{~nm}$ & $130 \mathrm{~nm}$ & $75 \mathrm{~nm}$ \\
\hline Contour length $l$ & $1890 \mathrm{~nm}$ & $1890 \mathrm{~nm}$ & $1890 \mathrm{~nm}$ \\
\hline End-to-end distance $R_{\mathrm{e}}$ & $170 \mathrm{~nm}$ & $170 \mathrm{~nm}$ & $170 \mathrm{~nm}$ \\
\hline Persistence length $l_{\mathrm{p}}$ & $21 \mathrm{~nm}$ & $4 \mathrm{~nm}$ & $1 \mathrm{~nm}$ \\
\hline Kuhn length $2 l_{\mathrm{p}}$ & $42 \mathrm{~nm}$ & $8 \mathrm{~nm}$ & $2 \mathrm{~nm}$ \\
\hline
\end{tabular}

\section{Time Scales}

In semidilute unentangled solutions, polymer chains undergo a crossover between two dynamic modes that occur at different time scales. The relaxations at length scales shorter than the correlation length $\xi$ are well-described by the Zimm model, up to the relaxation time of correlation blobs $\tau_{\xi} \sim \eta_{s} \xi^{3} / k_{B} T .^{2-4}$ At time scales larger than the relaxation time of a correlation blob $\left(t>\tau_{\xi}\right)$, polymers relax according to the Rouse model as a chain of correlation 
blobs. Chains move subdiffusively until the terminal relaxation time $\tau_{R} \sim \tau_{\xi}(N / g)^{2 \nu+1},{ }^{2,4}$ where $N / g$ represents the number of correlation blobs per chain and $\nu$ is the Flory exponent. We calculated particle diffusion times $\tau_{\mathrm{D}}=R_{\mathrm{NP}}^{2} / D$, where $D$ is the particle diffusivity at a given polymer concentration. Here, we summarize the characteristic relaxation time of a correlation blob, terminal relaxation time (Table S2), and particle diffusion times (Table S3).

Table S2: Estimations of correlation blob and terminal relaxation times for $2200 \mathrm{kDa}$ NaPSS solutions at $10^{-6} \mathrm{M}$ ionic strength.

\begin{tabular}{|c|c|c|}
\hline Polymer concentration $c / c^{*}$ & $\tau_{\xi}[\mathrm{ms}]$ & $\tau_{\mathrm{R}}[\mathrm{sec}]$ \\
\hline 1.5 & 0.957 & 0.342 \\
\hline 3 & 0.178 & 0.246 \\
\hline 5 & 0.067 & 0.203 \\
\hline 8 & 0.028 & 0.170 \\
\hline 12 & 0.013 & 0.146 \\
\hline 25 & 0.003 & 0.111 \\
\hline 60 & 0.001 & 0.080 \\
\hline
\end{tabular}

Table S3: Estimations of particle diffusion times for various particle sizes as a function of $2200 \mathrm{kDa}$ sodium polystyrene sulfonate concentrations at $10^{-6} \mathrm{M}$ ionic strength.

\begin{tabular}{|c|c|c|c|c|c|c|}
\hline & \multicolumn{5}{|c|}{$\tau_{\mathrm{D}}[\mathrm{sec}]$} \\
\hline Polymer concentration $c / c^{*}$ & $100 \mathrm{~nm}$ & $200 \mathrm{~nm}$ & $300 \mathrm{~nm}$ & $380 \mathrm{~nm}$ & $600 \mathrm{~nm}$ & $790 \mathrm{~nm}$ \\
\hline 2 & 0.001 & 0.011 & 0.043 & 0.088 & 0.354 & 0.757 \\
\hline 5 & 0.002 & 0.018 & 0.067 & 0.144 & 0.592 & 1.53 \\
\hline 10 & 0.003 & 0.040 & 0.113 & 0.194 & 1.20 & 2.84 \\
\hline 20 & 0.008 & 0.075 & 0.262 & 0.612 & 2.05 & 4.59 \\
\hline 30 & 0.013 & 0.137 & 0.363 & 0.840 & 2.90 & 6.00 \\
\hline 60 & 0.025 & 0.244 & 0.833 & 1.44 & 5.00 & 11.1 \\
\hline
\end{tabular}

\section{Dynamic Light Scattering (DLS)}

Dynamic light scattering measurements were conducted using a Brookhaven Instruments goniometer (BI-200SM, Brookhaven Instruments Corporation) equipped with an Avalanche photodiode detector (Brookhaven, BI-APD) and a digital correlator (Brookhaven, TurboCarr) and a laser (Mini-L30 Laser Source) with an excitation wavelength $\lambda=637.6 \mathrm{~nm}$. 
Glass vials were cleaned using acetone, water, and lens paper to minimize the scattering from contaminants on the surface. Dilute polymer solutions were prepared with a particle volume fraction of $10^{-5}$ for all samples. Scattered light was collected at angles $\theta=$ $60^{\circ}, 75^{\circ}, 90^{\circ}, 105^{\circ}$, and $120^{\circ}$, corresponding to wavevectors $q$ from 14 to $23 \mu \mathrm{m}^{-1}$, and for 60 seconds at 200 delay times ranging from $1 \mu$ s to $20 \mathrm{~s}$. From this data, the intensityintensity autocorrelation $g_{(2)}(\tau, q)=\langle I(t+\tau) I(t)\rangle /\langle I(t)\rangle^{2}$ functions were calculated. The field-field autocorrelation function $g_{(1)}(\tau, q)$ was obtained from the intensity-intensity autocorrelation function using the Siegert relation $g_{(2)}(\tau, q)=1+\beta\left[g_{(1)}(\tau, q)\right]^{2}+\varepsilon$, where $\varepsilon$ is noise, $g_{(1)}(\tau, q)=\exp (-t / \tau)$, and $\tau$ is the decay rate that represents the characteristic relaxation time of the system. Nanoparticle diffusivity was calculated using the relation $D=1 / \tau q^{2}$ and hydrodynamic radii of particles was estimated using Stokes-Einstein relationship $D_{\mathrm{SE}}=k_{\mathrm{B}} T / 6 \pi \eta R_{\mathrm{NP}}$, where the viscosity $\eta$ for each polymer concentration was determined using a capillary viscometer. We find that hydrodynamic radius of particles remains constant as polymer concentration increases (Figure S3), suggesting no adsorption of chains onto particle surfaces.
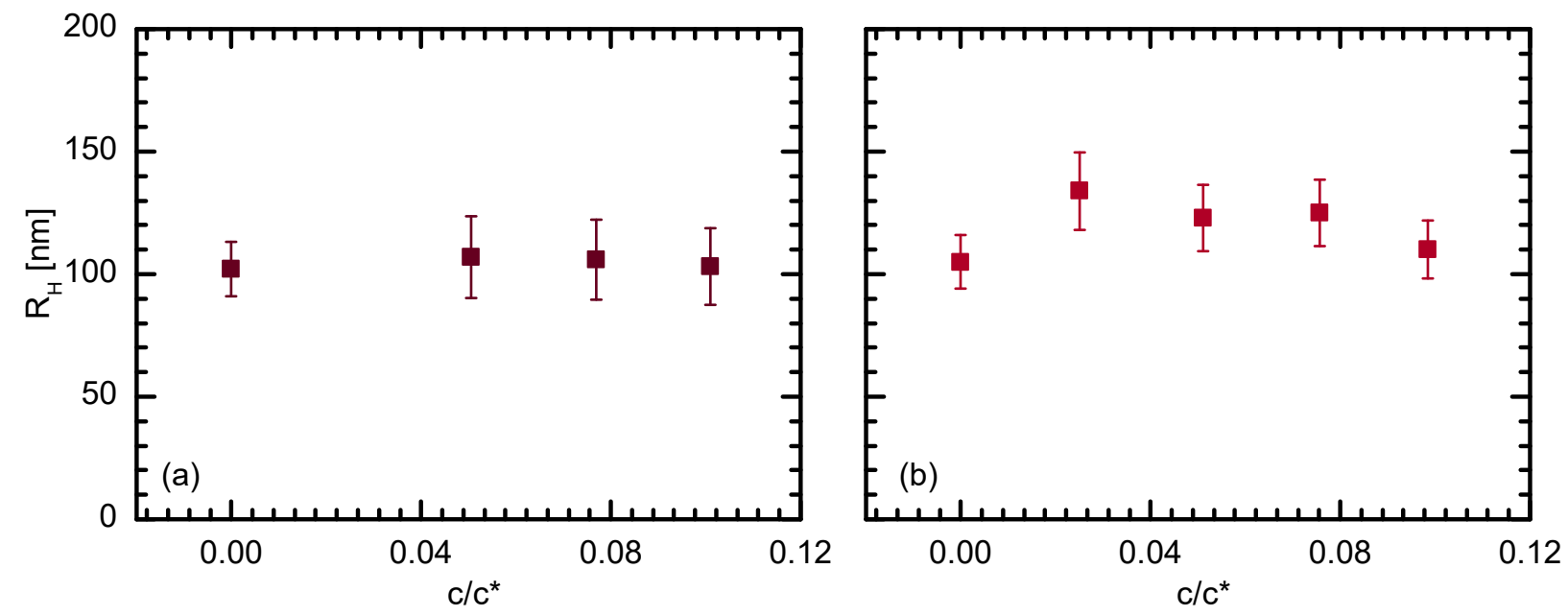

Figure S3: Hydrodynamic radius $R_{\mathrm{H}}$ as a function of normalized polymer concentration $c / c^{*}$ for $200 \mathrm{~nm}$ particles in solutions with (a) $10^{-6}$ and (b) $10^{-1} \mathrm{M}$ ionic strength. 


\section{Rheology}

Dynamic frequency sweep measurements were collected using a single-gap Couette cell on a Discovery Hybrid Rheometer (TA Instruments, HR-2). The cell has a diameter of 15 $\mathrm{mm}$, a bob diameter of $14 \mathrm{~mm}$, and a bob length of $42 \mathrm{~mm}$. We performed a dynamic strain sweep at angular frequencies $\omega=1,10$, and $100 \mathrm{rad} / \mathrm{s}$. The linear viscoelastic regime extended up to strains $\gamma=20 \%$. Storage $\left(G^{\prime}\right)$ and loss $\left(G^{\prime \prime}\right)$ moduli were measured at $10 \%$ strain as a function of angular frequency for NaPSS solutions. We eliminate any data points with generated torque values below $0.1 \mu \mathrm{N} \cdot \mathrm{m}$ or raw phase angle values above $175^{\circ}$ to meet the instrument's minimum criterion while minimizing the contribution of inertia to data collection. We find that terminal crossover is not reached within the experimented angular frequency range for all samples, suggesting that relaxations occur at very short time scales $<10 \mathrm{~ms}$ and an absence of entanglements in our solutions. In Figure S5, we present the viscosity as a function of shear rate for NaPSS solutions at $10^{-3}$ and $10^{-1} \mathrm{M}$ ionic strength. The viscosity is independent of shear rate, indicating that chains relax quickly in solutions. These results are consistent with the $10^{-6} \mathrm{M}$ solutions in the manuscript.
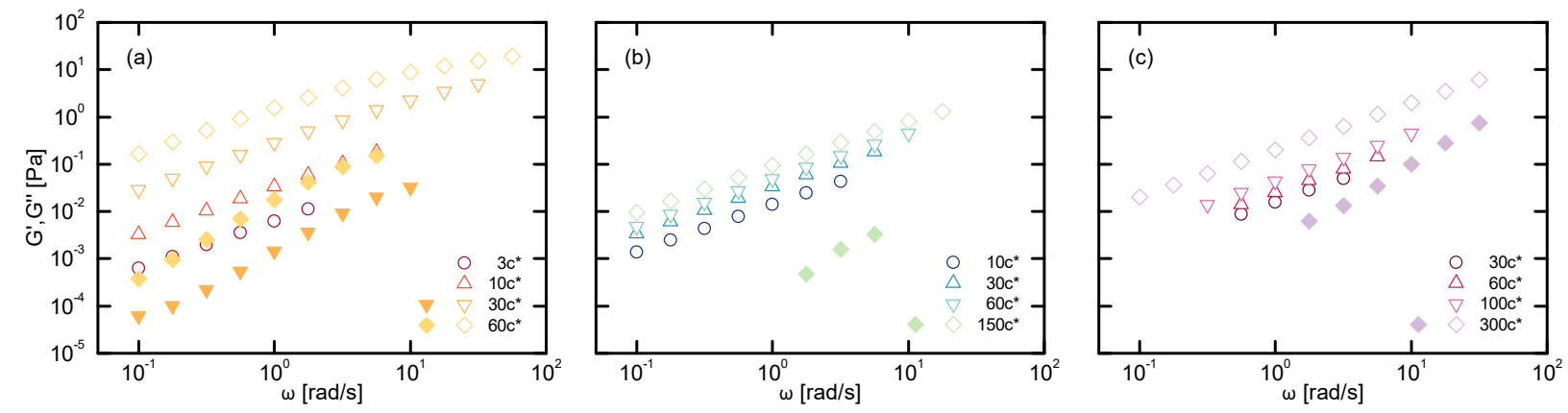

Figure S4: Storage $G^{\prime}$ (closed) and loss $G^{\prime \prime}$ (open) moduli as a function of angular frequency $\omega$ for (a) $10^{-1}$, (b) $10^{-3}$, and (c) $10^{-6} \mathrm{M}$ ionic strength solutions. 

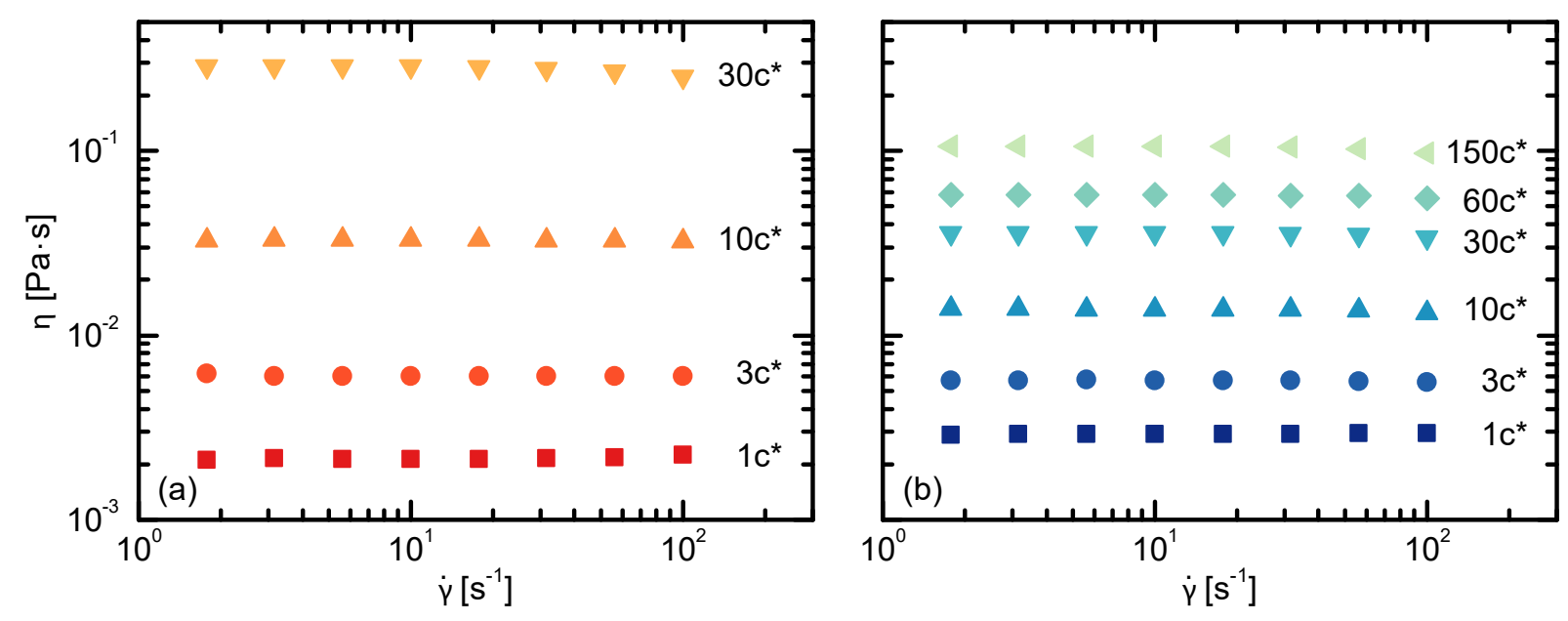

Figure S5: Viscosity $\eta$ for (a) $10^{-1}$ and (b) $10^{-3} \mathrm{M}$ ionic strength NaPSS solutions as a function of shear rate $\dot{\gamma}$. 


\section{Obstruction Models}

We examined the normalized diffusivities $D / D_{0}$ for $10^{-6} \mathrm{M}$ ionic strength solutions according to the proposed scaling for obstruction pictures. ${ }^{5-7}$ Here, we plot the data only for those particles for which the assumption for an immobile mesh is valid (i.e. $\tau_{R}>\tau_{D}$ ). We find that the model is not able to capture the observed dynamics.

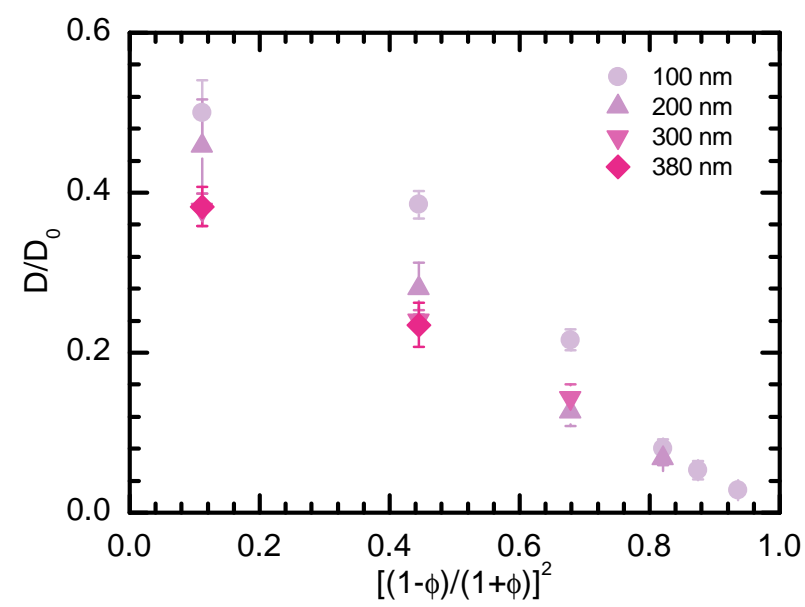

Figure S6: Relative diffusivity $D / D_{0}$ as a function of $\left(\frac{1-\phi}{1+\phi}\right)^{2}$.

\section{Hydrodynamic Models}

We examined the scaled diffusivities $D / D_{0}$ or $D_{0} / D$ for $10^{-6} \mathrm{M}$ ionic strength samples as a function of the proposed length scales of various hydrodynamic models, ${ }^{8-11}$ particle-polymer coupling theory, ${ }^{12}$ and depletion layer theory. ${ }^{13}$ None of the models cleanly collapse the dynamics across all particle sizes or polymer concentrations. 


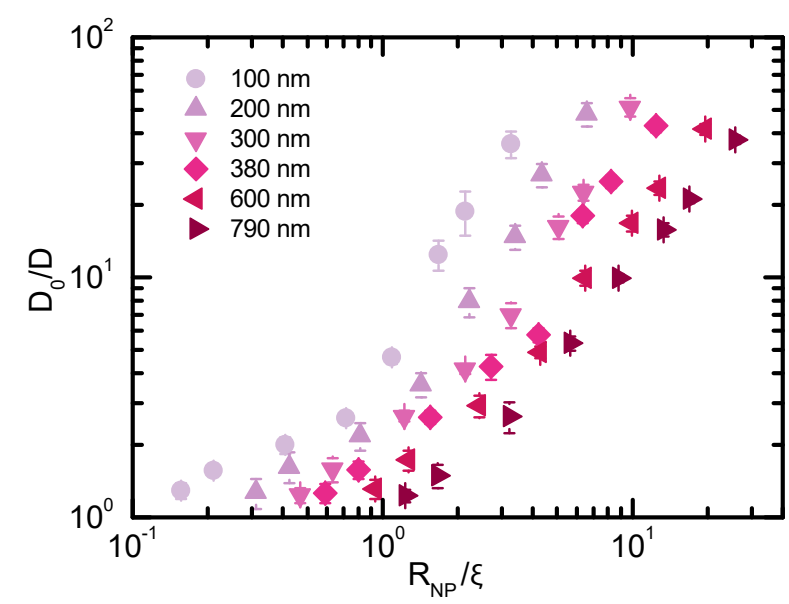

Figure S7: Relative diffusivity $D_{0} / D$ as a function of $R_{\text {Cheng }} / \xi$, where $R_{\text {Cheng }}=R_{\mathrm{NP}}$. This model does not collapse the data at any particle or polymer size. ${ }^{8}$

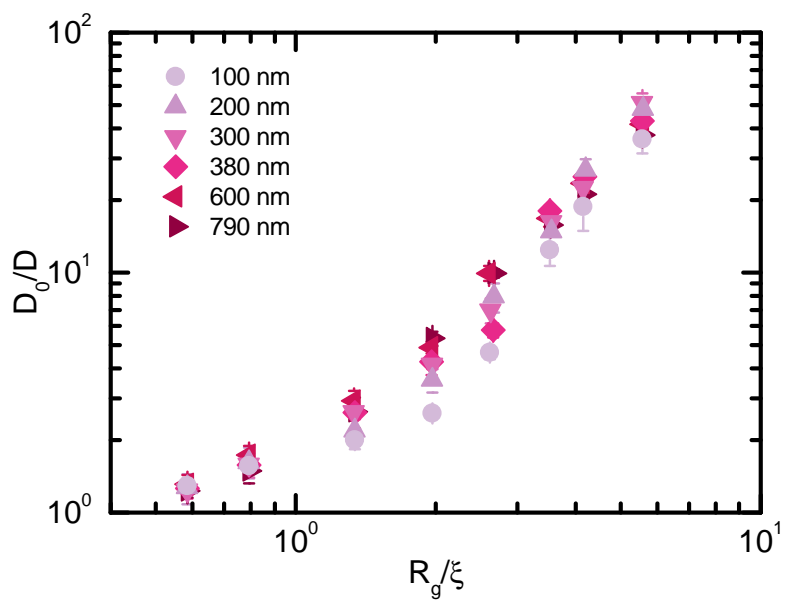

Figure S8: Relative diffusivity $D_{0} / D$ as a function of $R_{\mathrm{g}} / \xi$. The extent to which this model is able to collapse the data decreases as particles become smaller. The model successfully collapsed data for large $\left(R_{\mathrm{NP}}>R_{\mathrm{g}}\right)$ particles that follow SE behavior. ${ }^{9}$ 


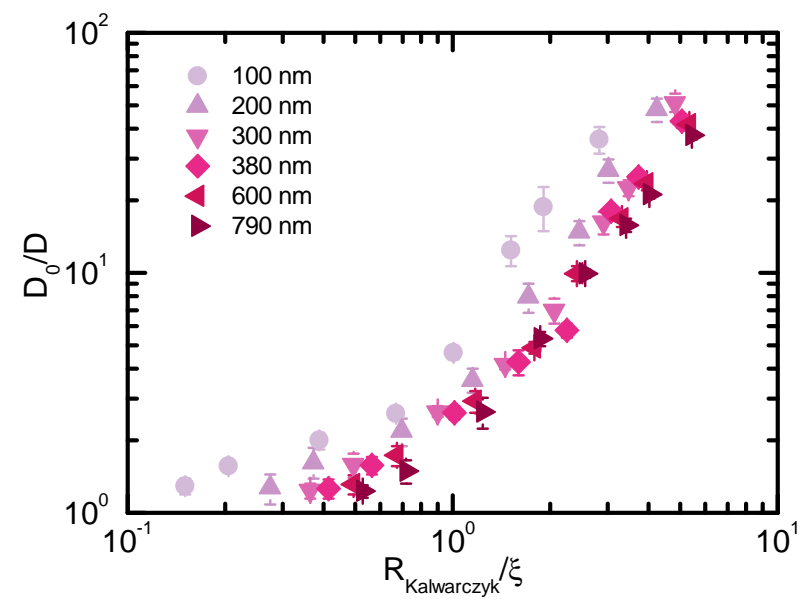

Figure S9: Relative diffusivity $D_{0} / D$ as a function of $R_{\text {Kalwarczyk }} / \xi$. The effective radius $R_{\text {Kalwarczyk }}$ is defined as $R_{\text {Kalwarczyk }}^{-2}=R_{\mathrm{NP}}^{-2}+R_{\mathrm{g}}^{-2}$. This model captures the dynamics of large particles but fails to collapse the dynamics of small particles onto the same curve. ${ }^{10}$

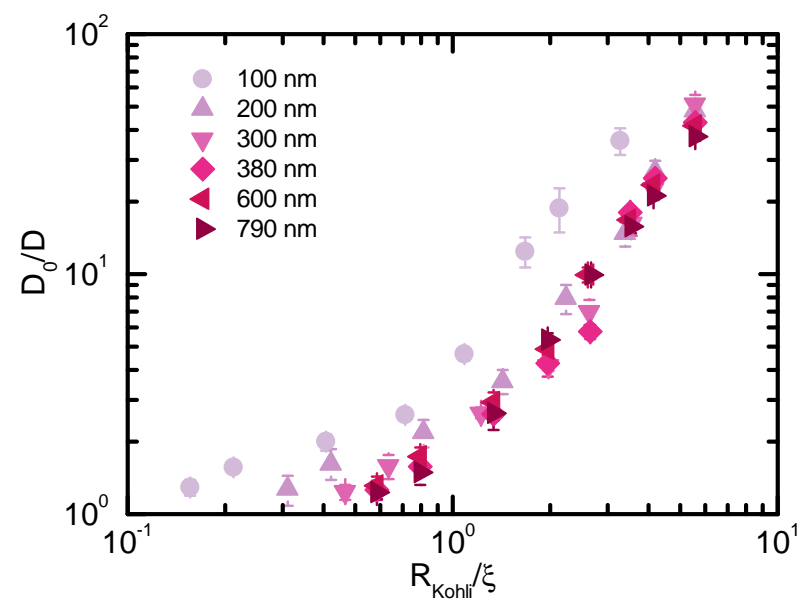

Figure S10: Relative diffusivity $D_{0} / D$ as a function of $R_{\text {Kohli }}$. The effective radius $R_{\text {Kohli is }}$ defined as $R_{\text {Kohli }}=R_{\mathrm{NP}}$ if $R_{\mathrm{NP}}<R_{\mathrm{g}}$ and $R_{\text {Kohli }}=R_{\mathrm{g}}$ if $R_{\mathrm{NP}}>R_{\mathrm{g}}$. The model does not capture the behavior of the $100 \mathrm{~nm}$ particles. ${ }^{11}$ 


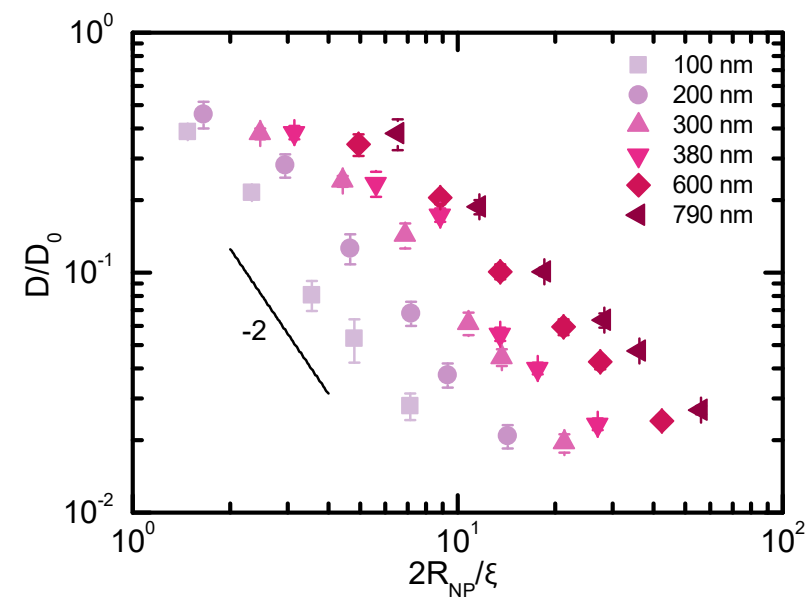

Figure S11: Relative diffusivity $D / D_{0}$ as a function of $2 R_{\mathrm{NP}} / \xi$. The coupling theory picture predicts intermediate size particles $\left(\xi<R_{\mathrm{NP}}<a\right.$, where $\xi$ is correlation length, and $a$ is the tube diameter) should collapse according to this scaling with a scaling exponent of -2 . The model is unable to collapse the data onto a master curve, although some data follow the predicted scaling -2 across limited ranges. ${ }^{12}$

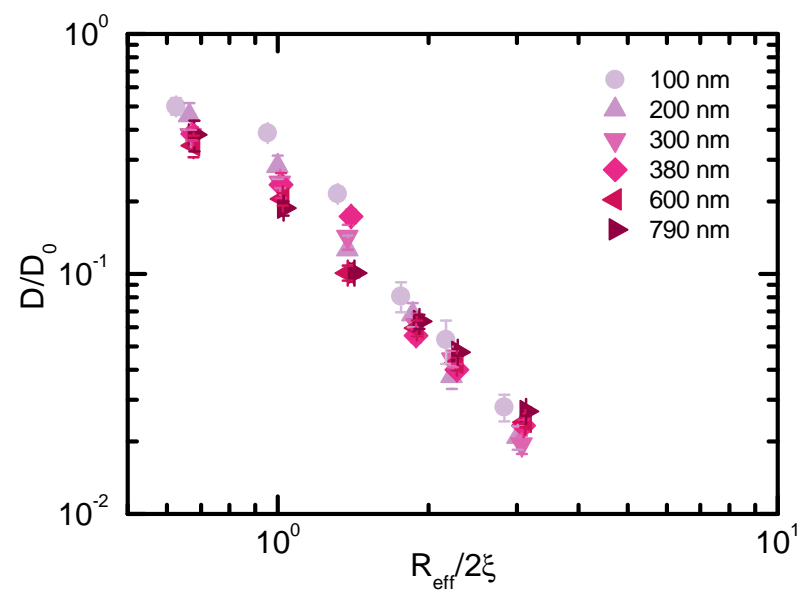

Figure S12: Relative diffusivity $D / D_{0}$ as a function of $2 R_{\text {eff }} / 2 \xi$. The effective radius $R_{\text {eff }}$ is defined as $R_{\text {eff }}=\sqrt{\frac{R_{\mathrm{g}}^{2}(2 R+2 d)^{2}}{R_{\mathrm{g}}^{2}+(2 R+2 d)^{2}}}$, where $d$ is the depletion layer thickness. This model predicts the dynamics at high polymer concentration, but we observe discrepancies in low concentration solutions. ${ }^{13}$ 


\section{Displacement Autocorrelations}

We calculate displacement autocorrelation functions to quantify the correlation between two successive displacements. The calculations are conducted according to

$$
\begin{gathered}
\Delta x(t)=x\left(t+\Delta t_{\text {int }}\right)-x(t) \\
C_{\mathrm{d}}^{*}(\tau)=<\Delta x(t+\tau) \Delta x(t)>
\end{gathered}
$$

where $x(t)$ represents the particle position at a time $t, \Delta t_{\text {int }}$ is the real time step for change of displacement, and $\tau$ is the correlation displacement step. Angular brackets denote the ensemble-average at multiple time origins. To compare sample to sample data more easily, we normalize the autocorrelation coefficient via

$$
C_{\mathrm{d}}(\tau)=\frac{C_{\mathrm{d}}^{*}(\tau)}{C_{\mathrm{d}}^{*}(0)}
$$

where $C_{\mathrm{d}}^{*}(0)$ represents the displacement autocorrelation value of the first displacement step. This choice normalizes the function such that at $t=0$ it has the value 1 for all samples. We observe more pronounced anticorrelation for small particles compared to large ones, suggesting that small particles experience caging-like effects with increasing polymer concentrations (Figure S13). 

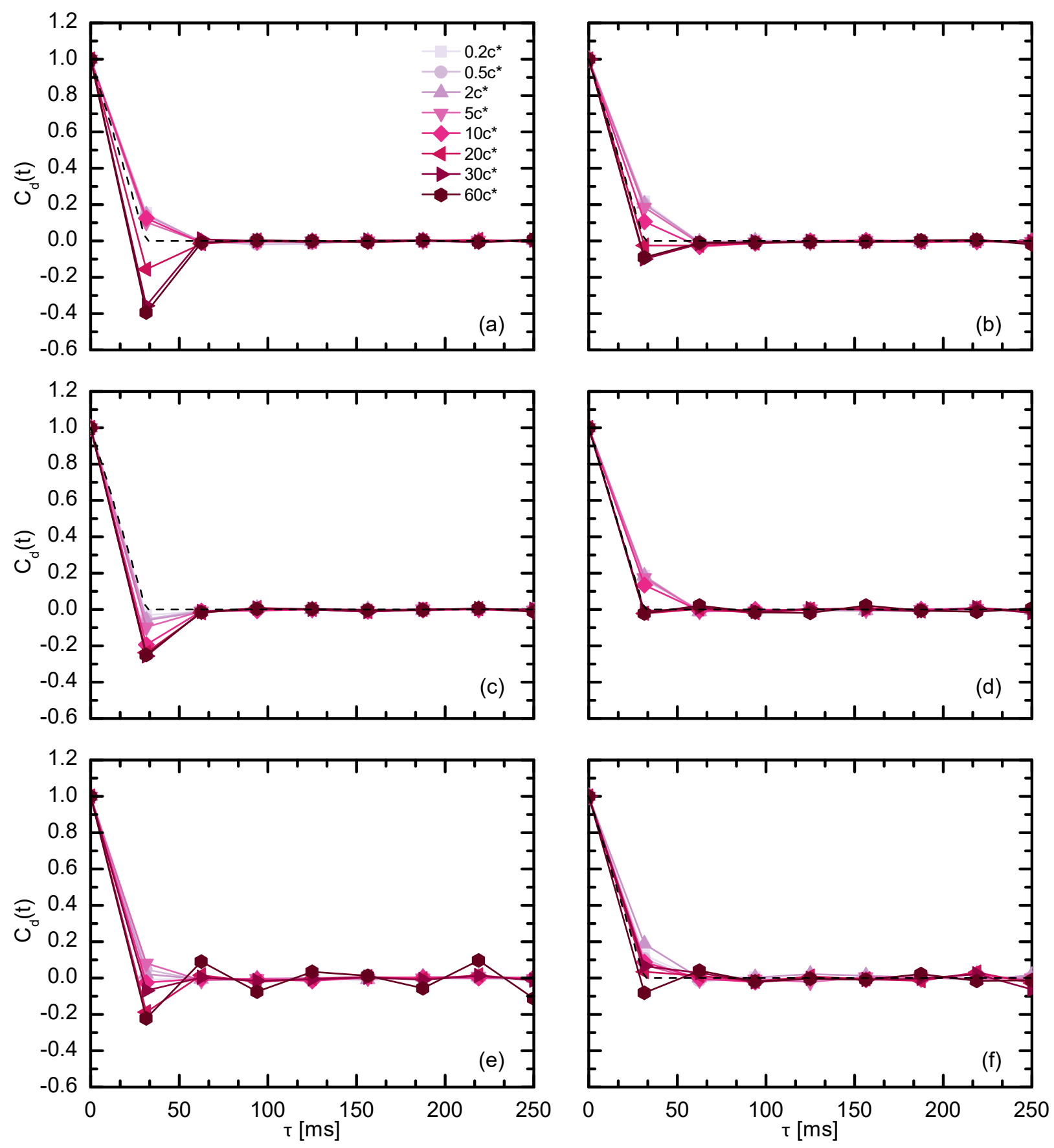

Figure S13: Normalized coefficient of displacement autocorrelation $C_{\mathrm{d}}(t)$ as a function of elapsed time $\tau$ for (a) 100, (b) 200, (c) 300, (d) 380, (e) 600, and (f) $790 \mathrm{~nm}$ particles in NaPSS solutions at $10^{-6} \mathrm{M}$ ionic strength. Dashed line represents the displacement autocorrelation of a Gaussian process. 


\section{References}

(1) Flory, P. J. Principles of Polymer Chemistry; Cornell University Press: Ithaca, NY, 1953.

(2) Colby, R. H. Structure and linear viscoelasticity of flexible polymer solutions: Comparison of polyelectrolyte and neutral polymer solutions. Rheol. Acta 2010, 49, 425-442.

(3) Zimm, B. H. Dynamics of Polymer Molecules in Dilute Solution: Viscoelasticity, Flow Birefringence and Dielectric Loss. J. Chem. Phys. 1956, 24, 269.

(4) Rubinstein, M.; Colby, R. H. Polymer Physics; Oxford University Press: New York, 2003.

(5) Mackie, J. S.; Meares, P. The diffusion of electrolytes in a cation-exchange resin membrane. II. Experimental. P. Proc. R. Soc. London, Ser. A 1955, 232, 510-518.

(6) Ogston, A. G. The spaces in a uniform random suspension of fibres. Trans. Faraday Soc. 1958, 54, 1754-1757.

(7) Ogston, A. G.; Preston, B. N.; Wells, J. D. on the Transport of Compact Particles Through Solutions of Chain-Polymers. Proc. R. Soc. Lond. A 1973, 333, 297-316.

(8) Cheng, Y.; Prud'homme, R. K.; Thomas, J. L. Diffusion of mesoscopic probes in aqueous polymer solutions measured by fluorescence recovery after photobleaching. Macromolecules 2002, 35, 8111-8121.

(9) Holyst, R.; Bielejewska, A.; Szymański, J.; Wilk, A.; Patkowski, A.; Gapiński, J.; Zywociński, A.; Kalwarczyk, T.; Kalwarczyk, E.; Tabaka, M.; Zibacz, N.; Wieczorek, S. A. Scaling form of viscosity at all length-scales in poly(ethylene glycol) solutions studied by fluorescence correlation spectroscopy and capillary electrophoresis. Phys. Chem. Chem. Phys. 2009, 11, 9025-9032. 
(10) Kalwarczyk, T.; Ziębacz, N.; Bielejewska, A.; Zaboklicka, E.; Koynov, K.; Szymański, J.; Wilk, A.; Patkowski, A.; Gapiński, J.; Butt, H.-J.; Hołyst, R. Comparative analysis of viscosity of complex liquids and cytoplasm of mammalian cells at the nanoscale. Nano Lett. 2011, 11, 2157-2163.

(11) Kohli, I.; Mukhopadhyay, A. Diffusion of nanoparticles in semidilute polymer solutions: Effect of different length scales. Macromolecules 2012, 45, 6143-6149.

(12) Cai, L.-H.; Panyukov, S.; Rubinstein, M. Mobility of Spherical Probe Objects in Polymer Liquids. Macromolecules 2011, 44, 7853-7863.

(13) Zȩibacz, N.; Wieczorek, S. A.; Kalwarczyk, T.; Fiałkowski, M.; Hołyst, R. Crossover regime for the diffusion of nanoparticles in polyethylene glycol solutions: Influence of the depletion layer. Soft Matter 2011, 7, 7181-7186. 